\title{
Dark Energy Survey Year 1 Results: Cosmological Constraints from Cluster Abundances, Weak Lensing, and Galaxy Correlations
}

C. To $\odot,{ }^{1,2,3,{ }^{*}}$ E. Krause, ${ }^{4,5, \dagger}$ E. Rozo,${ }^{5}$ H. Wu, ${ }^{6,7}$ D. Gruen, ${ }^{1,2,3}$ R. H. Wechsler, ${ }^{1,2,3}$ T. F. Eifler, ${ }^{4}$ E. S. Rykoff, ${ }^{2,3}$ M. Costanzi, ${ }^{8,9}$ M. R. Becker, ${ }^{10}$ G. M. Bernstein, ${ }^{11}$ J. Blazek, ${ }^{6,12}$ S. Bocquet, ${ }^{13}$ S. L. Bridle, ${ }^{14}$ R. Cawthon, ${ }^{15}$ A. Choi, ${ }^{6}$ M. Crocce ${ }^{16,17}$ C. Davis, ${ }^{2}$ J. DeRose, ${ }^{18,19}$ A. Drlica-Wagner, ${ }^{20,21,22}$ J. Elvin-Poole, ${ }^{6,23}$ X. Fang, ${ }^{4}$ A. Farahi, ${ }^{24}$ O. Friedrich ${ }^{25}$ M. Gatti, ${ }^{26}$ E. Gaztanaga, ${ }^{16,17}$ T. Giannantonio, ${ }^{27,25}$ W. G. Hartley, ${ }^{28,29,30}$ B. Hoyle, ${ }^{13,31,32}$ M. Jarvis, ${ }^{11}$ N. MacCrann, ${ }^{6,23}$ T. McClintock, ${ }^{5}$ V. Miranda, ${ }^{4}$ M. E. S. Pereira, ${ }^{24}$ Y. Park ${ }^{5}$ A. Porredon, ${ }^{6,16,17}$ J. Prat, ${ }^{20}$ M. M. Rau, ${ }^{33}$ A. J. Ross, ${ }^{6}$ S. Samuroff, ${ }^{33}$ C. Sánchez, ${ }^{11}$ I. Sevilla-Noarbe,${ }^{34}$ E. Sheldon, ${ }^{35}$ M. A. Troxel,${ }^{36}$ T. N. Varga ${ }^{31,32}$ P. Vielzeuf, ${ }^{26}$ Y. Zhang, ${ }^{21}$ J. Zuntz, ${ }^{37}$ T. M. C. Abbott, ${ }^{38}$ M. Aguena,${ }^{39,40}$ A. Amon, ${ }^{2}$ J. Annis, ${ }^{21}$ S. Avila, ${ }^{41}$ E. Bertin, ${ }^{42,43}$ S. Bhargava, ${ }^{44}$ D. Brooks,${ }^{29}$ D. L. Burke, ${ }^{2,3}$ A. Carnero Rosell, ${ }^{45,46}$ M. Carrasco Kind, ${ }^{47,48}$ J. Carretero, ${ }^{26}$ C. Chang, ${ }^{20,22}$ C. Conselice, ${ }^{14,49}$ L. N. da Costa, ${ }^{40,50}$ T. M. Davis, ${ }^{51}$ S. Desai, ${ }^{52}$ H. T. Diehl, ${ }^{21}$ J. P. Dietrich, ${ }^{13}$ S. Everett, ${ }^{19}$ A. E. Evrard,${ }^{53,24}$ I. Ferrero, ${ }^{54}$ B. Flaugher, ${ }^{21}$ P. Fosalba, ${ }^{16,17}$ J. Frieman, ${ }^{21,22}$ J. García-Bellido, ${ }^{41}$ R. A. Gruendl, ${ }^{47,48}$ G. Gutierrez, ${ }^{21}$ S. R. Hinton, ${ }^{51}$ D. L. Hollowood, ${ }^{19}$ K. Honscheid,,${ }^{6,23}$ D. Huterer, ${ }^{24}$ D. J. James,${ }^{55}$ T. Jeltema, ${ }^{19}$ R. Kron, ${ }^{21,22}$ K. Kuehn, ${ }^{56,57}$ N. Kuropatkin, ${ }^{21}$ M. Lima, ${ }^{39,40}$ M. A. G. Maia, ${ }^{40,50}$ J. L. Marshall, ${ }^{58}$ F. Menanteau, ${ }^{47,48}$ R. Miquel,,${ }^{59,26}$ R. Morgan, ${ }^{15}$ J. Muir, ${ }^{2}$ J. Myles, ${ }^{1}$ A. Palmese, ${ }^{21,22}$ F. Paz-Chinchón, ${ }^{27,48}$ A. A. Plazas, ${ }^{60}$ A. K. Romer, ${ }^{44}$ A. Roodman,,${ }^{2,3}$ E. Sanchez, ${ }^{34}$ B. Santiago, ${ }^{61,40}$ V. Scarpine, ${ }^{21}$ S. Serrano, ${ }^{16,17}$ M. Smith, ${ }^{62}$ E. Suchyta,${ }^{63}$ M. E. C. Swanson,${ }^{48}$ G. Tarle, ${ }^{24}$ D. Thomas, ${ }^{64}$ D. L. Tucker, ${ }^{21}$ J. Weller, ${ }^{31,32}$ W. Wester, ${ }^{21}$ and R. D. Wilkinson ${ }^{44}$

(DES Collaboration)

\footnotetext{
${ }^{1}$ Department of Physics, Stanford University, 382 Via Pueblo Mall, Stanford, California 94305, USA

${ }^{2}$ Kavli Institute for Particle Astrophysics \& Cosmology, P. O. Box 2450, Stanford University, Stanford, California 94305, USA

${ }^{3}$ SLAC National Accelerator Laboratory, Menlo Park, California 94025, USA

${ }^{4}$ Department of Astronomy/Steward Observatory, University of Arizona, 933 North Cherry Avenue, Tucson, Arizona 85721-0065, USA

${ }^{5}$ Department of Physics, University of Arizona, Tucson, Arizona 85721, USA

${ }^{6}$ Center for Cosmology and Astro-Particle Physics, The Ohio State University, Columbus, Ohio 43210, USA

${ }^{7}$ Department of Physics, Boise State University, Boise, Idaho 83725, USA

${ }^{8}$ INAF-Osservatorio Astronomico di Trieste, via G. B. Tiepolo 11, I-34143 Trieste, Italy

${ }^{9}$ Institute for Fundamental Physics of the Universe, Via Beirut 2, 34014 Trieste, Italy

${ }^{10}$ Argonne National Laboratory, 9700 South Cass Avenue, Lemont, Illinois 60439, USA

${ }^{11}$ Department of Physics and Astronomy, University of Pennsylvania, Philadelphia, Pennsylvania 19104, USA

${ }^{12}$ Institute of Physics, Laboratory of Astrophysics, École Polytechnique Fédérale de Lausanne (EPFL), Observatoire de Sauverny, 1290 Versoix, Switzerland

${ }^{13}$ Faculty of Physics, Ludwig-Maximilians-Universität, Scheinerstr. 1, 81679 Munich, Germany

${ }^{14}$ Jodrell Bank Center for Astrophysics, School of Physics and Astronomy, University of Manchester, Oxford Road, Manchester, M13 9PL, United Kingdom

${ }^{15}$ Physics Department, 2320 Chamberlin Hall, University of Wisconsin-Madison, 1150 University Avenue Madison, Wisconsin 53706-1390

${ }^{16}$ Institut d'Estudis Espacials de Catalunya (IEEC), 08034 Barcelona, Spain

${ }^{17}$ Institute of Space Sciences (ICE, CSIC), Campus UAB, Carrer de Can Magrans, s/n, 08193 Barcelona, Spain

${ }^{18}$ Department of Astronomy, University of California, Berkeley, 501 Campbell Hall, Berkeley, California 94720, USA

${ }^{19}$ Santa Cruz. Institute for Particle Physics, Santa Cruz, California 95064, USA

${ }^{20}$ Department of Astronomy and Astrophysics, University of Chicago, Chicago, Illinois 60637, USA

${ }^{21}$ Fermi National Accelerator Laboratory, P. O. Box 500, Batavia, Illinois 60510, USA

${ }^{22}$ Kavli Institute for Cosmological Physics, University of Chicago, Chicago, Illinois 60637, USA

${ }^{23}$ Department of Physics, The Ohio State University, Columbus, Ohio 43210, USA

${ }^{24}$ Department of Physics, University of Michigan, Ann Arbor, Michigan 48109, USA

${ }^{25}$ Kavli Institute for Cosmology, University of Cambridge, Madingley Road, Cambridge CB3 OHA, United Kingdom

${ }^{26}$ Institut de Física d'Altes Energies (IFAE), The Barcelona Institute of Science and Technology, Campus UAB, 08193 Bellaterra (Barcelona) Spain

${ }^{27}$ Institute of Astronomy, University of Cambridge, Madingley Road, Cambridge CB3 OHA, United Kingdom

${ }^{28}$ Département de Physique Théorique and Center for Astroparticle Physics, Université de Genève, 24 quai Ernest Ansermet, CH-1211 Geneva, Switzerland

${ }^{29}$ Department of Physics \& Astronomy, University College London, Gower Street, London, WC1E 6BT, United Kingdom
} 


\footnotetext{
${ }^{30}$ Department of Physics, ETH Zurich, Wolfgang-Pauli-Strasse 16, CH-8093 Zurich, Switzerland

${ }^{31}$ Max Planck Institute for Extraterrestrial Physics, Giessenbachstrasse, 85748 Garching, Germany

${ }^{32}$ Universitäts-Sternwarte, Fakultät für Physik, Ludwig-Maximilians Universität München, Scheinerstr. 1, 81679 München, Germany

${ }^{33}$ Department of Physics, Carnegie Mellon University, Pittsburgh, Pennsylvania 15312, USA

${ }^{34}$ Centro de Investigaciones Energéticas, Medioambientales y Tecnológicas (CIEMAT), Madrid, Spain

${ }^{35}$ Brookhaven National Laboratory, Bldg 510, Upton, New York 11973, USA

${ }^{36}$ Department of Physics, Duke University Durham, North Carolina 27708, USA

${ }^{37}$ Institute for Astronomy, University of Edinburgh, Edinburgh EH9 3HJ, United Kingdom

${ }^{38}$ Cerro Tololo Inter-American Observatory, NSF's National Optical-Infrared Astronomy Research Laboratory, Casilla 603, La Serena, Chile

${ }^{39}$ Departamento de Física Matemática, Instituto de Física, Universidade de São Paulo, CP 66318, São Paulo, SP, 05314-970, Brazil

${ }^{40}$ Laboratório Interinstitucional de e-Astronomia-LIneA, Rua Gal. José Cristino 77, Rio de Janeiro, RJ-20921-400, Brazil

${ }^{41}$ Instituto de Fisica Teorica UAM/CSIC, Universidad Autonoma de Madrid, 28049 Madrid, Spain

${ }^{42}$ CNRS, UMR 7095, Institut d'Astrophysique de Paris, F-75014, Paris, France

${ }^{43}$ Sorbonne Universités, UPMC Univ Paris 06, UMR 7095, Institut d'Astrophysique de Paris, F-75014, Paris, France

${ }^{44}$ Department of Physics and Astronomy, Pevensey Building, University of Sussex, Brighton, BN1 9QH, United Kingdom

${ }^{45}$ Instituto de Astrofisica de Canarias, E-38205 La Laguna, Tenerife, Spain

${ }^{46}$ Universidad de La Laguna, Dpto. Astrofsica, E-38206 La Laguna, Tenerife, Spain

${ }^{47}$ Department of Astronomy, University of Illinois at Urbana-Champaign, 1002 W. Green Street, Urbana, Illinois 61801, USA

${ }^{48}$ National Center for Supercomputing Applications, 1205 West Clark St., Urbana, Illinois 61801, USA

${ }^{49}$ University of Nottingham, School of Physics and Astronomy, Nottingham NG7 2RD, United Kingdom

${ }^{50}$ Observatório Nacional, Rua Gal. José Cristino 77, Rio de Janeiro, RJ-20921-400, Brazil

${ }^{51}$ School of Mathematics and Physics, University of Queensland, Brisbane, QLD 4072, Australia

${ }^{52}$ Department of Physics, IIT Hyderabad, Kandi, Telangana 502285, India

${ }^{53}$ Department of Astronomy, University of Michigan, Ann Arbor, Michigan 48109, USA

${ }^{54}$ Institute of Theoretical Astrophysics, University of Oslo. P.O. Box 1029 Blindern, NO-0315 Oslo, Norway

${ }^{55}$ Center for Astrophysics I Harvard \& Smithsonian, 60 Garden Street, Cambridge, Massachusetts 02138, USA

${ }^{56}$ Australian Astronomical Optics, Macquarie University, North Ryde, New South Wales 2113, Australia

${ }^{57}$ Lowell Observatory, 1400 Mars Hill Rd, Flagstaff, Arizona 86001, USA

${ }^{58}$ George P. and Cynthia Woods Mitchell Institute for Fundamental Physics and Astronomy, and Department of Physics and Astronomy, Texas A\&M University, College Station, Texas 77843, USA

${ }^{59}$ Institució Catalana de Recerca i Estudis Avanćats, E-08010 Barcelona, Spain

${ }^{60}$ Department of Astrophysical Sciences, Princeton University, Peyton Hall, Princeton, New Jersey 08544, USA

${ }^{61}$ Instituto de Física, UFRGS, Caixa Postal 15051, Porto Alegre, RS-91501-970, Brazil

${ }^{62}$ School of Physics and Astronomy, University of Southampton, Southampton, SO17 1BJ, United Kingdom

${ }^{63}$ Computer Science and Mathematics Division, Oak Ridge National Laboratory, Oak Ridge, Tennessee 37831

${ }^{64}$ Institute of Cosmology and Gravitation, University of Portsmouth, Portsmouth, PO1 3FX, United Kingdom
}

(Received 6 October 2020; revised 7 January 2021; accepted 23 February 2021; published 6 April 2021)

We present the first joint analysis of cluster abundances and auto or cross-correlations of three cosmic tracer fields: galaxy density, weak gravitational lensing shear, and cluster density split by optical richness. From a joint analysis $(4 \times 2 \mathrm{pt}+\mathrm{N})$ of cluster abundances, three cluster cross-correlations, and the auto correlations of the galaxy density measured from the first year data of the Dark Energy Survey, we obtain $\Omega_{m}=0.305_{-0.038}^{+0.055}$ and $\sigma_{8}=0.783_{-0.054}^{+0.064}$. This result is consistent with constraints from the DES-Y1 galaxy clustering and weak lensing two-point correlation functions for the flat $\nu \Lambda C D M$ model. Consequently, we combine cluster abundances and all two-point correlations from across all three cosmic tracer fields $(6 \times 2 \mathrm{pt}+\mathrm{N})$ and find improved constraints on cosmological parameters as well as on the cluster observable-mass scaling relation. This analysis is an important advance in both optical cluster cosmology and multiprobe analyses of upcoming wide imaging surveys.

DOI: 10.1103/PhysRevLett.126.141301

Introduction.-The standard flat $\Lambda \mathrm{CDM}$ model has been remarkably successful at describing a broad range of cosmological observations across the history of the Universe. However, a fundamental physics explanation of the two main constituents of this model—dark matter and dark energy_is still missing. This has inspired ambitious cosmic surveys that are testing the $\Lambda \mathrm{CDM}$ model with increasingly precise measurements of complementary cosmological probes [1].

Wide-field imaging surveys, such as the Dark Energy Survey (DES [2]), the Hyper-Suprime Cam Subaru Strategic Program (HSC [3]), and the Kilo Degree 
Survey (KiDS [4]), are one class of these cosmic surveys, which map the spatial distribution, shapes, and colors of millions of galaxies. These datasets enable a wide range of cosmological measurements [5-11]. Two of the most established cosmological probes are galaxy clustering and weak gravitational lensing. Analyses that include the autocorrelation of these two tracer fields as well as their cross correlation, galaxy-galaxy lensing, are referred to as $3 \times 2 \mathrm{pt}$ analyses and are emerging as a competitive cosmological test.

The abundances and spatial distribution of galaxy clusters provide another powerful probe of cosmic structure formation and expansion history [12]. The principal obstacle to robust cosmological inference from cluster abundances is an accurate calibration of the relation between cluster observables and cluster mass [13-17]. In this work, we combine three cluster related cross-correlations with galaxy clustering to calibrate this relation. We note that despite the use of galaxy clustering, the cosmological information in our combined analysis is driven by the cluster abundance data, with galaxy clustering breaking degeneracies between cosmology and the cluster observable-mass relation.

In this Letter, we first demonstrate the consistency between our cluster cosmology analysis $(4 \times 2 \mathrm{pt}+\mathrm{N})$, the $3 \times 2 \mathrm{pt}$ analysis, and other cluster cosmology analyses, in the context of the $\Lambda \mathrm{CDM}$ model with massive neutrinos $(\nu \Lambda \mathrm{CDM})$. We then present the first joint analysis, referred to as $6 \times 2 \mathrm{pt}+\mathrm{N}$, of galaxy clusters abundances and clustering, galaxy clustering, and weak gravitational lensing. In Fig. 1, we summarize the different components of the analysis. Our analysis uses the same set of systematics modeling, calibration procedures, and analysis pipeline

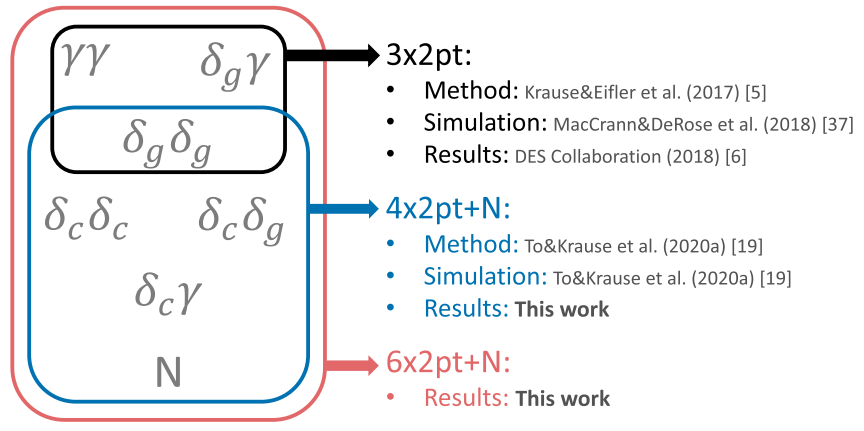

FIG. 1. Summary of the different components in this analysis and a nonexhaustive list of papers describing and validating our methodology. A more comprehensive list of relevant references can be found in Refs. [6,17] and references therein. The data in this Letter consist of cluster abundances $(N)$ and six two-point correlation functions derived from three cosmic tracer fields, namely, galaxy density $\left(\delta_{g}\right)$, weak gravitational lensing shear $(\gamma)$, and cluster density $\left(\delta_{c}\right)$. The correlation functions include cosmic shear $(\gamma \gamma)$, galaxy-galaxy lensing $\left(\delta_{g} \gamma\right)$, galaxy clustering $\left(\delta_{g} \delta_{g}\right)$, cluster-galaxy cross-correlation $\left(\delta_{c} \delta_{g}\right)$, cluster autocorrelation $\left(\delta_{c} \delta_{c}\right)$, and cluster lensing $\left(\delta_{c} \gamma\right)$. across all probes, and properly accounts for the covariance between the probes. We demonstrate that combining galaxy clusters and the $3 \times 2 \mathrm{pt}$ analysis improves both cosmological and cluster mass-observable relation constraints, compared to these individual analyses.

Data and measurement.-We measure galaxy density fields, weak gravitational lensing shear fields, and cluster density fields from the $1321 \mathrm{deg}^{2}$ of imaging data taken in the first season of the Dark Energy Survey [18] (DESY1). The measurement is based on procedures described in Ref. [19] using the DESY1 public catalogs [20]. These include the redMaGiC galaxy catalog [21] for the galaxy density field; the METACALIBRATION shape catalog [22] and BPZ photometric redshift (photo- $z$ ) catalog [23] for the weak gravitational lensing shear field; and the redMaPPer cluster catalog [24] for the cluster density field. To construct the galaxy density field, $\sim 650000$ redMaGiC galaxies over the redshift range $0.15<z<0.9$ are split into five redshift bins based on their photo- $z$ estimations. The weak gravitational lensing shear field is constructed based on $\sim 26 \times 10^{6}$ galaxies spanning the redshift range $0.2<z<1.3$, split into four redshift bins based on BPZ photo- $z$ estimation. For the cluster density fields, 4794 redMaPPer clusters are split into three redshift bins spanning the range $0.2<z<0.6$. The clusters are further split into four bins based on their richness $(\lambda)$, a cluster mass proxy defined as a weighted sum of the cluster redsequence member galaxies. The clusters span the richness range $20<\lambda<235$.

We measure six two-point correlations from the three cosmic tracer fields, as described in Fig. 1. The $3 \times 2 \mathrm{pt}$ correlations are the DESY1 public $3 \times 2$ pt data vector [25]. The cluster (cross-)correlations and cluster abundances are measured following procedures described in Ref. [19].

Modeling and inference.-We assume a Gaussian likelihood function as detailed below.

Covariance and model. The covariance matrix [26] is derived based on halo models [32,33] and is validated in Refs. [5,19]. The derivation and construction procedures are detailed in Ref. [19]. We relate the abundances of galaxy clusters to the halo mass function [34] assuming a power-law relation with log-normal scatter between the halo mass and cluster richness [19]. The three cosmic tracer fields are assumed to be linearly related to the matter density field, whose power spectrum is modeled using CLASS [35] and HALOFIT [36]. The model of cosmic shear and galaxy-galaxy lensing is described and validated in Refs. [5,37], while the model of $4 \times 2 \mathrm{pt}+\mathrm{N}$ is described and validated in Ref. [19] with modifications to the modeling of the effect of massive neutrinos [26]. Both the covariance matrix derivation and the model prediction are implemented in COSMOLIKE [33].

Analysis choices. We have designed our analysis to ensure robustness of the inferred result. Key analysis choices are summarized below. (i) Only large scale 
information is used.-Because of uncertainties of modeling baryonic effects, nonlinear relations between cosmic tracer fields and matter density fields, and random fluctuations of sparse tracers on small scales, we adopt conservative angular scale cuts on the two-point correlation functions. The scale cuts of $3 \times 2 \mathrm{pt}$ data vectors are defined and validated in Ref. [5]; the scale cuts of $4 \times 2 \mathrm{pt}+\mathrm{N}$ are defined and validated in Ref. [19]. (ii) The same set of parameters and priors are used in $3 \times 2 \mathrm{pt}, 4 \times 2 \mathrm{pt}+\mathrm{N}$, and $6 \times 2 \mathrm{pt}+\mathrm{N}$ analyses. - In addition to the six cosmological parameters in the $\nu \Lambda C D M$ model, we simultaneously sample over 26 nuisance parameters [26]. These include galaxy bias parameters (5), lens and source galaxy photo- $z$ biases (9), multiplicative shear biases (4), intrinsic alignment parameters (2), parameters describing the richness-mass relation (4), and parameters describing selection bias for clusters (2). For detailed descriptions of these nuisance parameters and the associated priors, we refer the readers to Refs. $[5,19,26]$. We note that we do not account for intrinsic alignments in the cluster lensing analysis. The effect is expected to be small [38] and was not included in the previous weak lensing analysis of the same sample [39]. In addition, in the cluster lensing model, we exclude bins where the maximum redshift of galaxy clusters is larger than the mean redshift of source galaxies. (iii) The analysis was done blindly. - Cosmological parameters were blinded by random shifts before the analysis choices were determined. We detail our blinding procedure in Ref. [26].

We use MultiNest [40] to generate Monte Carlo Markov Chain (MCMC) samples from the posterior. We find consistent results when using EMCEE [41].

Results and discussions.-Table I presents the cosmological parameter constraints from $3 \times 2 \mathrm{pt}, 4 \times 2 \mathrm{pt}+\mathrm{N}$, and $6 \times 2 \mathrm{pt}+\mathrm{N}$.

Cluster cosmology. We first compare our cosmological constraints $(4 \times 2 \mathrm{pt}+\mathrm{N})$ with cluster analyses in the

TABLE I. Summary of cosmological parameter constraints in the $\nu \Lambda \mathrm{CDM}$ model from three combinations of data vectors, as described in Fig. 1. The number reported is the 1D peak of the posterior and the asymmetric $68 \%$ confidence interval. Cells with no entries correspond to posteriors dominated by the priors. The last two rows summarize the goodness of fit for each data vector computed at the best-fit model.

\begin{tabular}{lcccc}
\hline \hline Parameter & $3 \times 2 \mathrm{pt}$ & $4 \times 2 \mathrm{pt}+\mathrm{N}$ & $6 \times 2 \mathrm{pt}+\mathrm{N}$ & Flat prior \\
\hline$\Omega_{m}$ & $0.297 \pm 0.036$ & $0.305_{-0.038}^{+0.055}$ & $0.276_{-0.026}^{+0.033}$ & {$[0.1,0.9]$} \\
$A_{s}\left(\times 10^{9}\right)$ & $2.15_{-0.34}^{+0.38}$ & $2.27_{-0.41}^{+0.57}$ & $2.08_{-0.31}^{+0.41}$ & {$[0.5,5]$} \\
$n_{s}$ & $\ldots$ & $\ldots$ & $\ldots$ & {$[0.87,1.07]$} \\
$\Omega_{b}$ & $\ldots$ & $\ldots$ & $\ldots$ & {$[0.03,0.07]$} \\
$\Sigma m_{\nu}[\mathrm{eV}]$ & $\ldots$ & $\ldots$ & $\ldots$ & {$[0.047,0.931]$} \\
$h$ & $\ldots$ & $\ldots$ & $\ldots$ & {$[0.55,0.91]$} \\
$\sigma_{8}$ & $0.771_{-0.054}^{+0.064}$ & $0.783_{-0.054}^{+0.064}$ & $0.802_{-0.048}^{+0.056}$ & Derived \\
$\chi^{2}$ (d.o.f) & $512(444)$ & $610(567)$ & $1054(992)$ & \\
$p$ value & 0.014 & 0.103 & 0.084 & \\
\hline \hline
\end{tabular}

literature. The result is shown in Fig. 2. According to the $Q_{\mathrm{DM}}$ tension metric [42], the $4 \times 2 \mathrm{pt}+\mathrm{N}$ constraints are consistent (Since no tension metric can guarantee consistency, we use the word "consistent" as a short expression of no significant inconsistency found by the tension metric throughout the Letter.) with most of the cluster cosmology analyses within $0.6 \sigma$, except for the constraints from a joint analysis of cluster abundances and weak lensing mass estimates in the DES-Y1 data [17] (hereafter called DES20). DES20 is in $2.9 \sigma$ tension with our $4 \times 2 \mathrm{pt}+\mathrm{N}$ analysis despite the fact that the two analyses share the same galaxy cluster and weak gravitational lensing shear catalogs. The main difference between $4 \times 2 \mathrm{pt}+\mathrm{N}$ and DES20 is that $4 \times 2 \mathrm{pt}+\mathrm{N}$ only uses large-scale information while the DES20 signal to noise is dominated by small-scale cluster lensing. We note that a similar tension has been found when comparing DES20 with a joint analysis of the DES cluster abundances and SPT-SZ multiwavelength data [43] (hereafter called $\mathrm{C} 20)$. In C20, the cluster mass-observable scaling relation is calibrated by cross matching the redMaPPer and SPT-SZ catalog (mean $\lambda=78$ ) and using the high-quality $\mathrm{x}$-ray and weak lensing follow-up data available for 121 SPT-SZ clusters to constrain the scaling relation [44-49]. Comparison between DES20, C20, and $4 \times 2 \mathrm{pt}+\mathrm{N}$ suggests that the tension between the DES20 analysis and other cluster cosmology analyses is likely due to unmodeled systematic artifacts in the weak lensing data of the redMaPPer clusters at small scales. This is consistent with the interpretation advanced by DES20. Alternatively, the low lensing signal observed for redMaPPer clusters may be

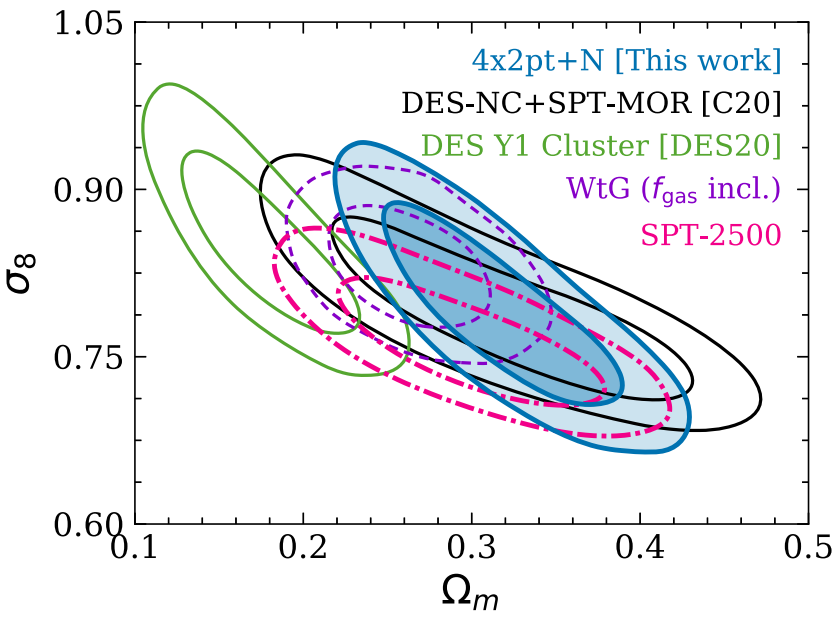

FIG. 2. Comparison of $\nu \Lambda C D M$ constraints on $\Omega_{\mathrm{m}}$ and $\sigma_{8}$ derived from $4 \times 2 \mathrm{pt}+\mathrm{N}$ (blue) and other cluster cosmology analyses in the literature: DES-Y1 joint analysis of cluster abundances and weak lensing mass estimates from Ref. [17] (green); a joint analysis of DES cluster abundances and SPT-SZ multiwavelength data from Ref. [43] (black); the Weighing the Giants study from Ref. [14] (purple); the SPT-2500 analysis from Ref. [15] (pink). Contours show $68 \%$ and $95 \%$ confidence levels. 


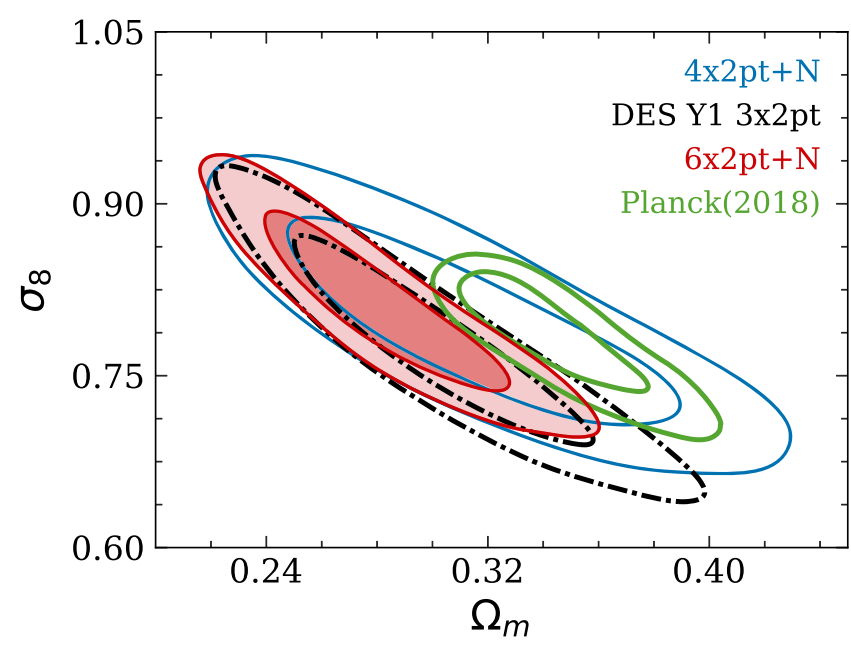

FIG. 3. $\nu \Lambda C D M$ constraints on $\Omega_{\mathrm{m}}$ and $\sigma_{8}$ from $3 \times 2 \mathrm{pt}$ (black), $4 \times 2 \mathrm{pt}+\mathrm{N}$ (blue), and their combination (red). For comparison, the green contours show constraints from the $\mathrm{CMB}$ at high redshift (Planck without lensing). Contours show 68\% and 95\% confidence levels.

related to the lensing-is-low problem for massive galaxies in the SDSS [50]. Should these two lensing anomalies be related, it is interesting to note that this anomaly disappears at the high mass end of the mass function. The resolution to the lensing anomaly at small scales remains unknown.

Systematics of redMaPPer clusters. Photometrically selected galaxy clusters are subject to two important systematics: projection effects $[17,51,52]$ and orientation biases [17,53]. These two systematics bias the observed galaxy and matter overdensities of the selected galaxy clusters relative to randomly selected halos of the same mass. On large scales these two effects manifest as a multiplicative bias factor $\left(b_{\text {sel }}\right)$ in the amplitude of the correlation functions, which can be sufficiently described by a power law in mass: $b_{\text {sel }}(M)=b_{s 0}(M / 5 \times$ $\left.10^{14} h^{-1} M_{\odot}\right)^{b_{s 1}}$ [19]. From the $6 \times 2 \mathrm{pt}+\mathrm{N}$ analysis, we obtain $b_{s 0}=1.15_{-0.09}^{+0.11}$ and $b_{s 1}=-0.029_{-0.062}^{+0.056}$. This result can be used to compare against future simulation-based estimates of these systematics.

Comparison of different cosmological probes in the Dark Energy Survey. Figure 3 shows a comparison between $3 \times 2 \mathrm{pt}$ and $4 \times 2 \mathrm{pt}+\mathrm{N}$. Here, before the analysis was unblinded, the tension metric was set to $Q_{\text {UDM }}[42,54]$, which compares the parameters from $3 \times 2 \mathrm{pt}$ and from its combination with $4 \times 2 \mathrm{pt}+\mathrm{N}$. According to $Q_{\mathrm{UDM}}$, the tension between $3 \times 2 \mathrm{pt}$ and $4 \times 2 \mathrm{pt}+\mathrm{N}$ is $0.024 \sigma$, indicating a strong consistency between galaxy clustering, weak gravitational lensing, and galaxy clusters in the context of the $\nu \Lambda C D M$ model. Given the demonstrated consistency between $3 \times 2 \mathrm{pt}$ and $4 \times 2 \mathrm{pt}+\mathrm{N}$, we proceed to perform a joint analysis of cluster abundances and all six two-point correlations derived from galaxy density fields, galaxy cluster density fields, and weak gravitational lensing shear fields. The constraints from this combination $(6 \times 2 \mathrm{pt}+\mathrm{N})$ are shown in Fig. 3. Our $6 \times 2 \mathrm{pt}+\mathrm{N}$ analysis leads to a $\sim 20 \%$ improvement on the constraints of $\Omega_{\mathrm{m}}$ relative to the $3 \times 2 \mathrm{pt}$ constraints.

Since DES only measures the matter distribution when the Universe is older than 10 billion years, it is interesting to compare our constraints to those derived from the early Universe as inferred from the Cosmic Microwave Background (CMB). Specifically, we compare our result with the prediction from the joint TT, EE, BB, TE likelihood measured by the Plank satellite [55], reanalyzed using the DES analysis choice of marginalizing over the unknown sum of neutrino masses [6]. The comparison is shown in Fig. 3. Despite the visual offset between the Planck $\nu \Lambda \mathrm{CDM}$ prediction and $6 \times 2 \mathrm{pt}+\mathrm{N}$, we find that the tension is at the level of $1.42 \sigma$, according to the tension

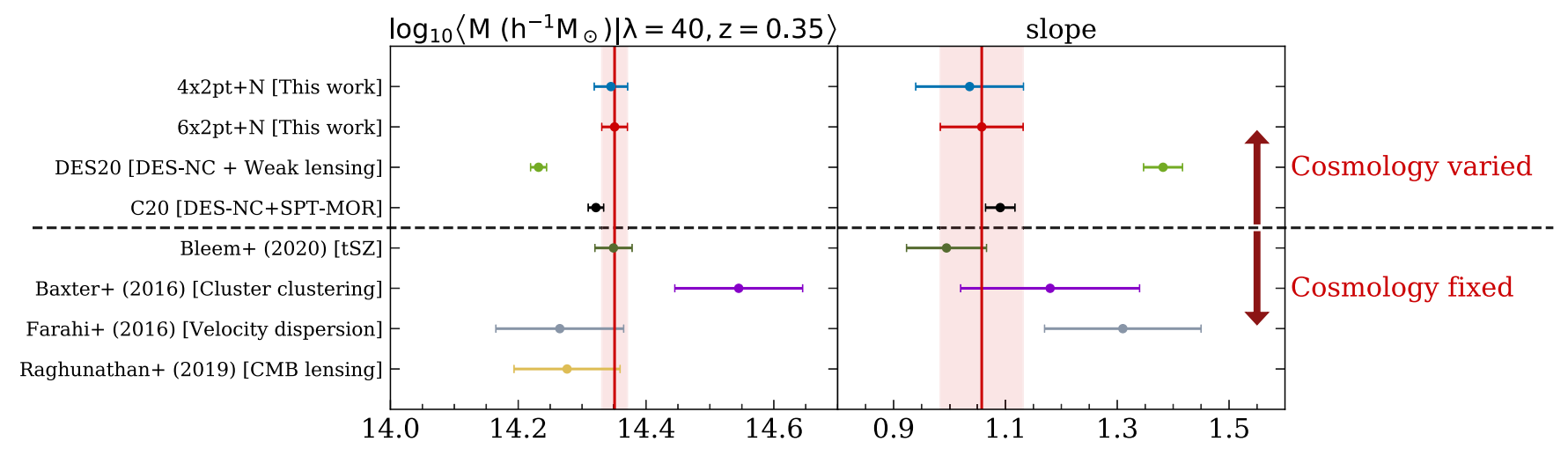

FIG. 4. Comparison of the predicted mean mass at richness $\lambda=40$ and redshift $z=0.35$ and the slope of the richness scaling relation from this Letter (blue and red) with results in literature: a joint analysis of number counts and weak lensing mass estimates [17] (light green); a joint analysis of DES cluster abundances and SPT-SZ multiwavelength data [43] (black); SZ scaling relation [61] (dark green); autocorrelations of galaxy clusters [62] (purple); velocity dispersion [63] (gray); and CMB lensing [64] (brown). Error bars show $68 \%$ confidence intervals. The slope is unconstrained in Ref. [64]. We note that Refs. [17,43] marginalize over cosmological parameters while Refs. [61-64] fix cosmological parameters. 
metric [56]. The consistency between $6 \times 2 \mathrm{pt}+\mathrm{N}$ and Planck is strong confirmation of the validity of the $\nu \Lambda \mathrm{CDM}$ model. Built on many previous works-Refs. $[6,17]$ and references therein-Fig. 3 presents the first joint analysis of galaxy clustering, galaxy lensing, and galaxy cluster abundance and clustering. This is an important milestone in multiprobe analyses of imaging surveys.

Mean mass of redMaPPer clusters. A precise measurement of cluster masses is important, for cosmological exploitation of cluster samples as well as for astrophysical studies involving galaxy clusters [57-60]. From $4 \times 2 \mathrm{pt}+$ $\mathrm{N}$ and $6 \times 2 \mathrm{pt}+\mathrm{N}$ analyses, we can derive the mean mass of the redMaPPer clusters and its dependence on the richness. The result is shown in Fig. 4 and the calculation is detailed in Ref. [26]. The $6 \times 2 \mathrm{pt}+\mathrm{N}$ analysis yields a $\sim 20 \%$ improvement on the constraints of mean cluster masses and their richness dependency compared to $4 \times 2 \mathrm{pt}+\mathrm{N}$. From the $6 \times 2 \mathrm{pt}+\mathrm{N}$ analysis, the mean mass of redMaPPer clusters at $z=0.35$ is constrained as

$$
\left\langle M_{200 \mathrm{~m}} \mid \lambda\right\rangle=10^{14.351 \pm 0.020}\left(\frac{\lambda}{40}\right)^{1.058 \pm 0.074} h^{-1} M_{\odot},
$$

where $M_{200 \mathrm{~m}}$ is the mass enclosed within a sphere in which the mean matter density is equal to 200 times the mean matter density of the Universe. In Fig. 4, we compare our constraints with results in the literature and find that our constraints are competitive. In terms of the consistency between different methods, we caution the reader that constraints on the mean mass and the slope of the massrichness relation might change by up to $15 \%$ and $10 \%$, respectively, due to assumptions about the modeling of projection effects [43]. Homogenization of projectioneffect modeling is beyond the scope of this work.

Conclusions and outlook.-In this Letter, we present the first joint analysis of cluster abundances and six two-point correlation functions derived from three cosmic tracer fields: galaxy density, weak gravitational lensing shear, and cluster density. Our findings can be summarized as follows: (i) Despite the surprising results of the DES-Y1 cluster abundances analysis [17], our multiprobe cluster cosmology approach finds consistent results compared with other cluster cosmology analyses and other cosmological probes in DES. This is likely a consequence of our analysis being restricted to large scales only. This result, together with C20 [43], suggests that the modeling of small-scale cluster lensing for low mass optically selected clusters is the likely culprit behind the surprising results in Ref. [17]. (ii) We find that combining galaxy clusters with galaxy clustering and weak gravitational lensing improves both cosmological constraints and constraints on the mean mass of galaxy clusters by $\sim 20 \%$, compared to results from galaxy clustering and weak gravitational lensing. (iii) The combined cosmological constraint from DES is consistent with Planck at the $1.4 \sigma$ level in the context of the $\nu \Lambda \mathrm{CDM}$ model. (iv) Combining galaxy clusters with galaxy clustering and weak gravitational lensing provides a precise constraint on the mean mass of galaxy clusters and its richness dependence.

In the near future, we expect a $\sim 40 \%$ improvement in cosmological constraints for $4 \times 2 \mathrm{pt}+\mathrm{N}$ from the analysis of the first three years of data from the Dark Energy Survey, mostly due to the increased survey area. This improvement will be followed by significant additional improvements from upcoming wide imaging surveys in the 2020s [65-67]. The analysis presented in this Letter is an important step towards fully realizing the potential of these richer and larger datasets.

This Letter has gone through internal review by the DES Collaboration. This work was supported in part by the U.S. Department of Energy contract to SLAC National Accelerator Laboratory, under Contract No. DE-AC0276 SF00515 (C. H., D. G., R. W.) including a Panofsky Fellowship awarded to D. G. E. K. is supported by the Department of Energy Grant No. DE-SC0020247. E. R. is supported by DOE Grants No. DE-SC0015975 and No. DE-SC0009913, and by NSF Grant No. 2009401. E. R. also acknowledges funding from the Cottrell Scholar program of the Research Corporation for Science Advancement. H.W. is supported by NSF Grant No. AST-1516997. Some of the computing for this project was performed on the Sherlock cluster at Stanford. We would like to thank KIPAC, Stanford University, and the Stanford Research Computing Center for providing computational resources and support that contributed to these research results. Funding for the DES Projects has been provided by the DOE and NSF(USA), MEC/MICINN/ MINECO(Spain), STFC(UK), HEFCE(UK). NCSA (UIUC), KICP(U. Chicago), CCAPP(Ohio State), MIFPA(Texas A\&M), CNPQ, FAPERJ, FINEP (Brazil), DFG(Germany), and the Collaborating Institutions in the Dark Energy Survey. The Collaborating Institutions are Argonne Lab, UC Santa Cruz, University of Cambridge, CIEMAT-Madrid, University of Chicago, University College London, DES-Brazil Consortium, University of Edinburgh, ETH Zürich, Fermilab, University of Illinois, ICE (IEEC-CSIC), IFAE Barcelona, Lawrence Berkeley Lab, LMU München and the associated Excellence Cluster Universe, University of Michigan, NFS's NOIRLab, University of Nottingham, Ohio State University, University of Pennsylvania, University of Portsmouth, SLAC National Lab, Stanford University, University of Sussex, Texas A\&M University, and the OzDES Membership Consortium. Based in part on observations at Cerro Tololo Inter-American Observatory at NSFs NOIRLab (NOIRLab Prop. ID 2012B-0001; PI: J. Frieman), which is managed by the Association of Universities for Research in Astronomy (AURA) under a cooperative agreement with the National Science Foundation. The DES Data Management System is 
supported by the NSF under Grants No. AST-1138766 and No. AST-1536171. The DES participants from Spanish institutions are partially supported by MICINN under Grants ESP2017-89838, PGC2018-094773, PGC2018102021, SEV-2016-0588, SEV-2016-0597, and MDM2015-0509, some of which include ERDF funds from the European Union. I. F. A. E. is partially funded by the CERCA program of the Generalitat de Catalunya. Research leading to these results has received funding from the European Research Council under the European Union's Seventh Framework Program (FP7/2007-2013) including ERC Grant Agreements 240672, 291329, and 306478. We acknowledge support from the Brazilian Instituto Nacional de Ciência e Tecnologia (INCT) do e-Universo (CNPq Grant No. 465376/2014-2). This manuscript has been authored by Fermi Research Alliance, LLC under Contract No. DE-AC02-07CH11359 with the U.S. Department of Energy, Office of Science, Office of High Energy Physics.

*chto@stanford.edu

${ }^{\dagger}$ krausee@arizona.edu

[1] D. H. Weinberg, M. J. Mortonson, D. J. Eisenstein, C. Hirata, A. G. Riess, and E. Rozo, Phys. Rep. 530, 87 (2013).

[2] Dark Energy Survey, https://www.darkenergysurvey.org/

[3] Subaru Strategic Program, http://www.naoj.org/Projects/ HSC/HSCProject.html

[4] Kilo Degree Survey, http://www.astro-wise.org/projects/ KIDS/

[5] E. Krause, T. F. Eifler, J. Zuntz, O. Friedrich, M. A. Troxel, S. Dodelson, J. Blazek, L. F. Secco, N. MacCrann, E. Baxter et al., arXiv:1706.09359.

[6] T. M. C. Abbott, F. B. Abdalla, A. Alarcon, J. Aleksić, S. Allam, S. Allen, A. Amara, J. Annis, J. Asorey, S. Avila et al., Phys. Rev. D 98, 043526 (2018).

[7] C. Hikage, M. Oguri, T. Hamana, S. More, R. Mandelbaum, M. Takada, F. Köhlinger, H. Miyatake, A. J. Nishizawa, H. Aihara et al., Publ. Astron. Soc. Jpn. 71, 43 (2019).

[8] T. Hamana, M. Shirasaki, S. Miyazaki, C. Hikage, M. Oguri, S. More, R. Armstrong, A. Leauthaud, R. Mandelbaum, H. Miyatake et al., Publ. Astron. Soc. Jpn. 72, 16 (2020).

[9] E. van Uitert, B. Joachimi, S. Joudaki, A. Amon, C. Heymans, F. Köhlinger, M. Asgari, C. Blake, A. Choi, T. Erben et al., Mon. Not. R. Astron. Soc. 476, 4662 (2018).

[10] S. Joudaki, C. Blake, A. Johnson, A. Amon, M. Asgari, A. Choi, T. Erben, K. Glazebrook, J. Harnois-Déraps, C. Heymans et al., Mon. Not. R. Astron. Soc. 474, 4894 (2018).

[11] C. Heymans, T. Tröster, M. Asgari, C. Blake, H. Hildebrandt, B. Joachimi, K. Kuijken, C.-A. Lin, A. G. Sánchez, J. L. van den Busch et al., arXiv:2007.15632.

[12] S. W. Allen, A. E. Evrard, and A. B. Mantz, Annu. Rev. Astron. Astrophys. 49, 409 (2011).

[13] A. von der Linden, M. T. Allen, D. E. Applegate, P. L. Kelly, S. W. Allen, H. Ebeling, P. R. Burchat, D. L. Burke,
D. Donovan, R. G. Morris et al., Mon. Not. R. Astron. Soc. 439, 2 (2014).

[14] A. B. Mantz, A. von der Linden, S. W. Allen, D. E. Applegate, P. L. Kelly, R. G. Morris, D. A. Rapetti, R. W. Schmidt, S. Adhikari, M. T. Allen et al., Mon. Not. R. Astron. Soc. 446, 2205 (2015).

[15] S. Bocquet, J. P. Dietrich, T. Schrabback, L. E. Bleem, M. Klein, S. W. Allen, D. E. Applegate, M. L. N. Ashby, M. Bautz, M. Bayliss et al., Astrophys. J. 878, 55 (2019).

[16] M. Costanzi, E. Rozo, M. Simet, Y. Zhang, A. E. Evrard, A. Mantz, E. S. Rykoff, T. Jeltema, D. Gruen, S. Allen et al., Mon. Not. R. Astron. Soc. 488, 4779 (2019).

[17] T. M. C. Abbott, M. Aguena, A. Alarcon, S. Allam, S. Allen, J. Annis, S. Avila, D. Bacon, K. Bechtol, A. Bermeo et al., Phys. Rev. D 102, 023509 (2020).

[18] A. Drlica-Wagner, I. Sevilla-Noarbe, E. S. Rykoff, R. A. Gruendl, B. Yanny, D. L. Tucker, B. Hoyle, A. Carnero Rosell, G. M. Bernstein, K. Bechtol et al., Astrophys. J. Suppl. Ser. 235, 33 (2018).

[19] C. To, E. Krause, E. Rozo, H. Wu, D. Gruen, J. DeRose, E. S. Rykoff, R. H. Wechsler, M. R. Becker, M. Costanzi et al., Mon. Not. R. Astron. Soc. 502, 4093 (2021).

[20] https://des.ncsa.illinois.edu/releases/y1a1/key-catalogs

[21] E. Rozo, E. S. Rykoff, A. Abate, C. Bonnett, M. Crocce, C. Davis, B. Hoyle, B. Leistedt, H. V. Peiris, R. H. Wechsler et al., Mon. Not. R. Astron. Soc. 461, 1431 (2016).

[22] J. Zuntz, E. Sheldon, S. Samuroff, M. A. Troxel, M. Jarvis, N. MacCrann, D. Gruen, J. Prat, C. Sánchez, A. Choi et al., Mon. Not. R. Astron. Soc. 481, 1149 (2018).

[23] B. Hoyle, D. Gruen, G. M. Bernstein, M. M. Rau, J. De Vicente, W. G. Hartley, E. Gaztanaga, J. DeRose, M. A. Troxel, C. Davis et al., Mon. Not. R. Astron. Soc. 478, 592 (2018).

[24] E. S. Rykoff, E. Rozo, M. T. Busha, C. E. Cunha, A. Finoguenov, A. Evrard, J. Hao, B. P. Koester, A. Leauthaud, B. Nord et al., Astrophys. J. 785, 104 (2014).

[25] https://des.ncsa.illinois.edu/releases/y1a1/key-products

[26] See Supplemental Material at http://link.aps.org/supplemental/ 10.1103/PhysRevLett.126.141301 for details of our blinding strategy, unblinding tests, simulated likelihood analyses, derivation of the covariance matrix adopted in this analysis, modelings of massive neutrinos, full cosmological and nuisance parameters, detailed derivations of the cluster mean mass-richness relation, which includes Refs. [27-31].

[27] M. Costanzi, F. Villaescusa-Navarro, M. Viel, J.-Q. Xia, S. Borgani, E. Castorina, and E. Sefusatti, J. Cosmol. Astropart. Phys. 12 (2013) 012.

[28] F. Villaescusa-Navarro, F. Marulli, M. Viel, E. Branchini, E. Castorina, E. Sefusatti, and S. Saito, J. Cosmol. Astropart. Phys. 03 (2014) 011.

[29] E. Castorina, E. Sefusatti, R. K. Sheth, F. VillaescusaNavarro, and M. Viel, J. Cosmol. Astropart. Phys. 02 (2014) 049.

[30] E. S. Rykoff, E. Rozo, D. Hollowood, A. Bermeo-Hernand ez, T. Jeltema, J. Mayers, A. K. Romer, P. Rooney, A. Saro, C. Vergara Cervantes et al., Astrophys. J. Suppl. Ser. 224, 1 (2016).

[31] J. Elvin-Poole, M. Crocce, A. J. Ross, T. Giannantonio, E. Rozo, E. S. Rykoff, S. Avila, N. Banik, J. Blazek, S. L. Bridle et al., Phys. Rev. D 98, 042006 (2018). 
[32] A. Cooray and R. Sheth, Phys. Rep. 372, 1 (2002).

[33] E. Krause and T. Eifler, Mon. Not. R. Astron. Soc. 470, 2100 (2017).

[34] J. L. Tinker, B. E. Robertson, A. V. Kravtsov, A. Klypin, M. S. Warren, G. Yepes, and S. Gottlöber, Astrophys. J. 724, 878 (2010).

[35] D. Blas, J. Lesgourgues, and T. Tram, J. Cosmol. Astropart. Phys. 07 (2011) 034.

[36] R. Takahashi, M. Sato, T. Nishimichi, A. Taruya, and M. Oguri, Astrophys. J. 761, 152 (2012).

[37] N. MacCrann, J. DeRose, R. H. Wechsler, J. Blazek, E. Gaztanaga, M. Crocce, E. S. Rykoff, M. R. Becker, B. Jain, E. Krause et al., Mon. Not. R. Astron. Soc. 480, 4614 (2018).

[38] C. Sifón, H. Hoekstra, M. Cacciato, M. Viola, F. Köhlinger, R. F. J. van der Burg, D. J. Sand, and M. L. Graham, Astron. Astrophys. 575, A48 (2015).

[39] T. McClintock, T. N. Varga, D. Gruen, E. Rozo, E. S. Rykoff, T. Shin, P. Melchior, J. DeRose, S. Seitz, J. P. Dietrich et al., Mon. Not. R. Astron. Soc. 482, 1352 (2019).

[40] F. Feroz, M. P. Hobson, and M. Bridges, Mon. Not. R. Astron. Soc. 398, 1601 (2009).

[41] D. Foreman-Mackey, D. W. Hogg, D. Lang, and J. Goodman, Publ. Astron. Soc. Pac. 125, 306 (2013).

[42] M. Raveri and W. Hu, Phys. Rev. D 99, 043506 (2019).

[43] M. Costanzi, A. Saro, S. Bocquet, T. M. C. Abbott, M. Aguena, S. Allam, A. Amara, J. Annis, S. Avila, D. Bacon et al., Phys. Rev. D 103, 043522 (2021).

[44] L. E. Bleem, B. Stalder, T. de Haan, K. A. Aird, S. W. Allen, D. E. Applegate, M. L. N. Ashby, M. Bautz, M. Bayliss, B. A. Benson et al., Astrophys. J. Suppl. Ser. 216, 27 (2015).

[45] T. Schrabback, M. Schirmer, R. F. J. van der Burg, H. Hoekstra, A. Buddendiek, D. Applegate, M. Bradač, T. Eifler, T. Erben, M. D. Gladders et al., Astron. Astrophys. 610, A85 (2018).

[46] J. P. Dietrich, S. Bocquet, T. Schrabback, D. Applegate, H. Hoekstra, S. Grandis, J. J. Mohr, S. W. Allen, M. B. Bayliss, B. A. Benson et al., Mon. Not. R. Astron. Soc. 483, 2871 (2019).

[47] M. McDonald, B. A. Benson, A. Vikhlinin, B. Stalder, L. E. Bleem, T. de Haan, H. W. Lin, K. A. Aird, M. L. N. Ashby, M. W. Bautz et al., Astrophys. J. 774, 23 (2013).

[48] M. McDonald, S. W. Allen, M. Bayliss, B. A. Benson, L. E. Bleem, M. Brodwin, E. Bulbul, J. E. Carlstrom, W. R. Forman, J. Hlavacek-Larrondo et al., Astrophys. J. 843, 28 (2017).

[49] S. Bocquet, J. P. Dietrich, T. Schrabback, L. E. Bleem, M. Klein, S. W. Allen, D. E. Applegate, M. L. N. Ashby, M. Bautz, M. Bayliss et al., Astrophys. J. 878, 55 (2019).
[50] A. Leauthaud, S. Saito, S. Hilbert, A. Barreira, S. More, M. White, S. Alam, P. Behroozi, K. Bundy, J. Coupon et al., Mon. Not. R. Astron. Soc. 467, 3024 (2017).

[51] M. Costanzi, E. Rozo, E. S. Rykoff, A. Farahi, T. Jeltema, A. E. Evrard, A. Mantz, D. Gruen, R. Mandelbaum, J. DeRose et al., Mon. Not. R. Astron. Soc. 482, 490 (2019).

[52] T. Sunayama, Y. Park, M. Takada, Y. Kobayashi, T. Nishimichi, T. Kurita, S. More, M. Oguri, and K. Osato, Mon. Not. R. Astron. Soc. 496, 4468 (2020).

[53] H.-Y. Wu et al. (to be published).

[54] M. Raveri, G. Zacharegkas, and W. Hu, Phys. Rev. D 101, 103527 (2020).

[55] N. Aghanim, Y. Akrami, M. Ashdown, J. Aumont, C. Baccigalupi, M. Ballardini, A. J. Banday, R. B. Barreiro, N. Bartolo et al. (Planck Collaboration), Astron. Astrophys. 641, A6 (2020).

[56] Y. Park and E. Rozo, Mon. Not. R. Astron. Soc. 499, 4638 (2020).

[57] G. W. Pratt, M. Arnaud, A. Biviano, D. Eckert, S. Ettori, D. Nagai, N. Okabe, and T. H. Reiprich, Space Sci. Rev. 215, 25 (2019).

[58] T. Shin, S. Adhikari, E. J. Baxter, C. Chang, B. Jain, N. Battaglia, L. Bleem, S. Bocquet, J. DeRose, D. Gruen et al., Mon. Not. R. Astron. Soc. 487, 2900 (2019).

[59] C.-H. To, R. M. Reddick, E. Rozo, E. Rykoff, and R. H. Wechsler, Astrophys. J. 897, 15 (2020).

[60] N. Gupta, M. Pannella, J. J. Mohr, M. Klein, E. S. Rykoff, J. Annis, S. Avila, F. Bianchini, D. Brooks, E. Buckley-Geer et al., Mon. Not. R. Astron. Soc. 494, 1705 (2020).

[61] L. E. Bleem, S. Bocquet, B. Stalder, M. D. Gladders, P. A. R. Ade, S. W. Allen, A. J. Anderson, J. Annis, M. L. N. Ashby, J.E. Austermann et al., Astrophys. J. Suppl. Ser. 247, 25 (2020).

[62] E. J. Baxter, E. Rozo, B. Jain, E. Rykoff, and R. H. Wechsler, Mon. Not. R. Astron. Soc. 463, 205 (2016).

[63] A. Farahi, A. E. Evrard, E. Rozo, E. S. Rykoff, and R. H. Wechsler, Mon. Not. R. Astron. Soc. 460, 3900 (2016).

[64] S. Raghunathan, S. Patil, E. Baxter, B. A. Benson, L. E. Bleem, T. L. Chou, T. M. Crawford, G. P. Holder, T. McClintock, C. L. Reichardt et al., Astrophys. J. 872, 170 (2019).

[65] R. Mandelbaum, T. Eifler, R. Hložek, T. Collett, E. Gawiser, D. Scolnic, D. Alonso, H. Awan, R. Biswas et al. (The LSST Dark Energy Science Collaboration), arXiv:1809.01669.

[66] L. Amendola, S. Appleby, A. Avgoustidis, D. Bacon, T. Baker, M. Baldi, N. Bartolo, A. Blanchard, C. Bonvin, S. Borgani et al., Living Rev. Relativity 21, 2 (2018).

[67] T. Eifler, H. Miyatake, E. Krause, C. Heinrich, V. Miranda, C. Hirata, J. Xu, S. Hemmati, M. Simet, P. Capak et al., arXiv:2004.05271. 